\title{
Leveraging Genetic Reports and Electronic Health Records for the Prediction of Primary Cancers: Algorithm Development and Validation Study
}

Nansu Zong ${ }^{1}$, PhD; Victoria $\mathrm{Ngo}^{2}, \mathrm{PhD}$; Daniel J Stone ${ }^{1}$, BSc; Andrew Wen ${ }^{1}, \mathrm{MSc}$; Yiqing Zhao ${ }^{1}, \mathrm{PhD}$; Yue $\mathrm{Yu}^{1}$, PhD; Sijia Liu ${ }^{1}$, PhD; Ming Huang ${ }^{1}$, PhD; Chen Wang ${ }^{1}$, PhD; Guoqian Jiang ${ }^{1}$, MD, PhD

\footnotetext{
${ }^{1}$ Department of Health Sciences Research, Mayo Clinic, Rochester, MN, United States

${ }^{2}$ University of California Davis Health, Sacramento, CA, United States
}

\section{Corresponding Author:}

Guoqian Jiang, MD, PhD

Department of Health Sciences Research

Mayo Clinic

200 First Street

Rochester, MN

United States

Phone: 14803018000

Email: Jiang.Guoqian@mayo.edu

\section{Abstract}

Background: Precision oncology has the potential to leverage clinical and genomic data in advancing disease prevention, diagnosis, and treatment. A key research area focuses on the early detection of primary cancers and potential prediction of cancers of unknown primary in order to facilitate optimal treatment decisions.

Objective: This study presents a methodology to harmonize phenotypic and genetic data features to classify primary cancer types and predict cancers of unknown primaries.

Methods: We extracted genetic data elements from oncology genetic reports of 1011 patients with cancer and their corresponding phenotypical data from Mayo Clinic's electronic health records. We modeled both genetic and electronic health record data with HL7 Fast Healthcare Interoperability Resources. The semantic web Resource Description Framework was employed to generate the network-based data representation (ie, patient-phenotypic-genetic network). Based on the Resource Description Framework data graph, Node2vec graph-embedding algorithm was applied to generate features. Multiple machine learning and deep learning backbone models were compared for cancer prediction performance.

Results: With 6 machine learning tasks designed in the experiment, we demonstrated the proposed method achieved favorable results in classifying primary cancer types (area under the receiver operating characteristic curve [AUROC] $96.56 \%$ for all 9 cancer predictions on average based on the cross-validation) and predicting unknown primaries (AUROC $80.77 \%$ for all 8 cancer predictions on average for real-patient validation). To demonstrate the interpretability, 17 phenotypic and genetic features that contributed the most to the prediction of each cancer were identified and validated based on a literature review.

Conclusions: Accurate prediction of cancer types can be achieved with existing electronic health record data with satisfactory precision. The integration of genetic reports improves prediction, illustrating the translational values of incorporating genetic tests early at the diagnosis stage for patients with cancer.

(JMIR Med Inform 2021;9(5):e23586) doi: 10.2196/23586

\section{KEYWORDS}

genetic reports; electronic health records; predicting primary cancers; Fast Healthcare Interoperability Resources; FHIR; Resource Description Framework; RDF 


\section{Introduction}

Cancer is the second leading cause of death worldwide [1]. The health burden of cancer in the United States is substantial [2,3], with approximately 1.8 million new diagnoses and an estimated 600,000 deaths in 2020 alone [4]. Despite the advances in characterizing oncogenic mutations in the past few decades, overcoming the consequences of cellular self-renewal and neoplastic transformation remains a challenge in cancer therapy research [5]. Therefore, continued discoveries in causes, treatment, and management are needed to further the knowledge and understanding of this collection of related diseases [6].

Modern gene technology has provided an opportunity to identify certain gene mutations associated with increased cancer risk. Approximately $5 \%$ to $10 \%$ of all cancer diagnoses are linked to cancer predisposition syndromes [7-9]. Major syndromes of cancer disposition affecting adults include breast, ovarian, prostate, gastric, and pancreatic cancer [7]. Precision medicine initiatives call for the leveraging of clinical and genomic data to not only screen for cancers but also to help monitor cancer progression and guide therapy options [10]. Clinicians can facilitate early screening critical for risk assessment and surveillance [8]. If cancer is detected at an early stage, survival rates tend to be significantly higher than those for cancers diagnosed at an advanced stage [11-13]. Nash et al [11] cite figures as drastic as $90 \%$ survival for early ovarian cancer detection compared to only 5\% survival with advanced stage detection, as an example. The utilization of genetic tests in diagnosing primary cancer also becomes critical when the symptoms and the physical exams suggest unspecified cancer known as cancer of unknown primary [14]. Cancer of unknown primary accounts for $3 \%$ to $5 \%$ of all tumors [15]. The prediction of the primary cancer of cancer of unknown primary can significantly increase our current knowledge of metastasis and benefit the treatment of patients with cancer of unknown primary.

The implementation and adoption of health information technology have given frontline clinicians access to a large repository of longitudinal clinical data collected during health care encounters [16,17]. Medical insight and clinical decision making rely heavily upon access to these data from electronic health records. Artificial intelligence techniques, such as machine learning methods, are promising for finding patterns and discovering associations in health care data to help predict diseases [18]. Improved predictions can be made by integrating diverse types of digital data in patients' charts, which include diagnosis codes, clinical notes, laboratory test results, and treatment data [19].

As demand grows for genetic testing from patients and as genomic data continue to be incorporated into electronic health records, there is a need to study how genetic reports, along with electronic health record data, can be leveraged to predict cancers. Conventional computational methods for predictive models are based on features extracted from diverse data sources, known as bag of features [20]. The features in these models are treated independently, and the potential connections and patterns among the features cannot be fully explored to serve the prediction. A network-based data model can be used to represent the association between data models with edges, and the potential patterns are embedded in the topological structure of the network. Predictions from network-based data representations have achieved promising results in diverse biomedical areas, such as drug-target prediction [21] and patient clustering [22]. Representing correlations among phenotypic and genetic data elements through network-based data modeling shows great potential in cancer prediction.

The objective of this study was to harmonize phenotypic and genetic features for accurate and explainable cancer prediction, specifically: (1) developing a network-based framework with standard health care data exchange frameworks, the HL7 Fast Healthcare Interoperability Resources (FHIR) [23] and the Resource Description Framework (RDF) for graph-based data representations, (2) employing a state-of-the-art graph embedding algorithm, Node2vec [24], to obtain features for machine learning and deep learning models, and (3) implementing the proposed method with a collection of genetic reports of patients with cancer and the corresponding phenotypic data from Mayo Clinic's electronic health record systems and comprehensive experiments.

\section{Methods}

\section{Preliminary}

FHIR is a standardized data framework designed for data exchange between different medical centers to enable information to be captured as it is generated, significantly simplifying population and real-time updates of predefined data models $[23,25]$. The FHIR specification defines a set of granular clinical concepts and resources to provide standard data infrastructure to support implementations [23]. FHIR-based data models are built upon combinations of these resources and a set of attributes with value types. The common attributes (eg, identifier) and unique attributes (eg, bodySite) in a resource are used to facilitate data modeling. Common data types (eg, String and CodeableConcepts) are used to constrain the attribute based on an adaptation of clinically related ontologies, such as SNOMED CT [26], LOINC [27], and International Statistical Classification of Diseases ninth (ICD-9) and tenth revisions (ICD-10) [28].

RDF is a general metadata or data model that defines concepts and web-resources based on a variety of syntax notations and data serialization formats [29]. Inherited from the classical conceptual modeling approaches, RDF utilizes the expressions to form triples, subject-predicate-object, to model data elements (eg, web resources). Specifically, in this study, the subject denotes the clinical data elements (eg, patients), and the predicate denotes a relationship between 2 data elements.

\section{Framework}

We proposed a network-based framework (Figure 1) that represented cancer data using the FHIR standard and RDF to facilitate the cancer prediction process. Five types of data sources extracted from the electronic health record-genetic information, lab tests, diagnosis, medication, and family historical records-were represented with FHIR resources and 
converted to the RDF-based representation. A graph-embedding algorithm, Node2vec, was used to provide a vectorial representation of nodes in the resulting network along with bag of features to form the features for the classification models.

Figure 1. A network-based framework for cancer prediction based on Fast Healthcare Interoperability Resources and Resource Description Framework.

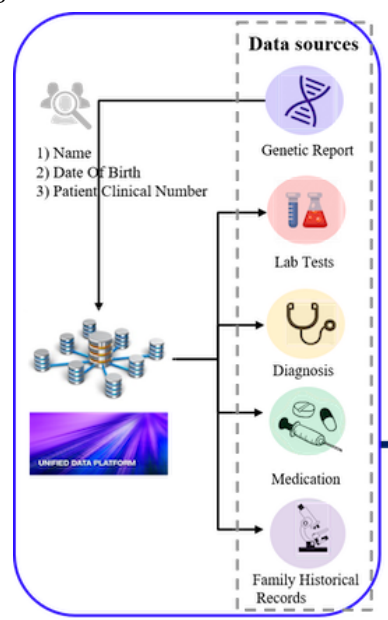

Data Preprocessing

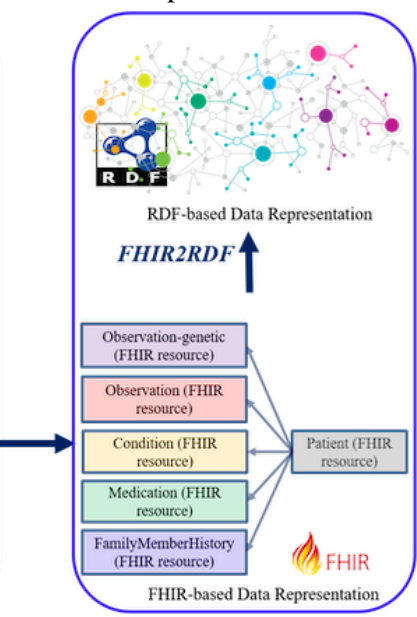

Data Modeling

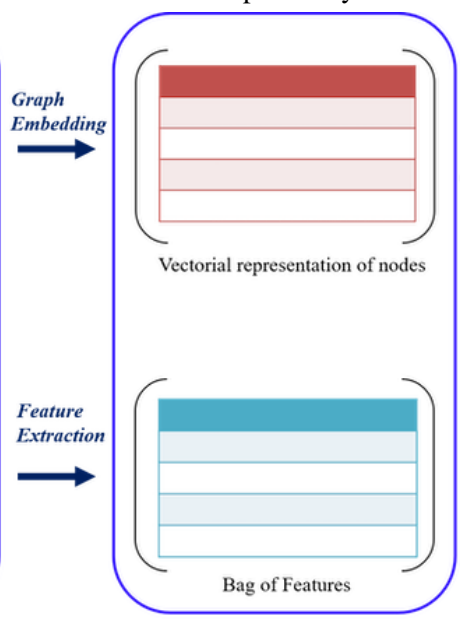

Feature Generation

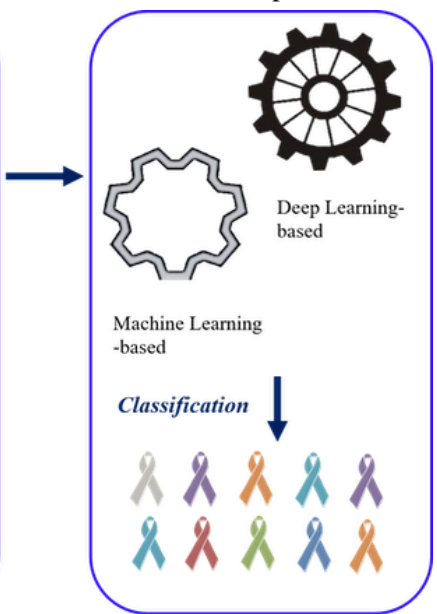

Cancer Prediction

\section{Data Preprocessing}

Genetic data were extracted from 1011 aggregated anonymized genetic test results (Foundation Medicine Inc), including microsatellite instability and tumor mutational burden. Medical record data elements related to laboratory results, diagnoses, medications, and family histories were extracted from approximately 515,000 billing encounters $(666,000$ electronic health record encounters) retrieved from a Mayo Clinic clinical data warehouse of [30]. We integrated genetic and electronic health record data by mapping patient information based on 3 data elements: patient clinic number, names (first and last name), and date of birth. Lab tests, diagnosis, medication, and family historical records were searched based on the mapped patients.
We used natural language processing to normalize the names and values. For diagnosis and medication, all diseases and medications were represented with standardized names encoded by ICD-9 [31] and RxNorm [32] codes. For lab tests, we represented all the tests with standard names encoded by LOINC [27]. For family historical records, each record was processed by a pipeline (NLP2FHIR [33]), where the medical concepts were identified and normalized using cTAKES [34], MedXN [35], and MedTime [36]. We encoded the diseases from family historical records using ICD-9 codes. To build the data set utilized for the cancer prediction, all the records within the billing circle related to the target cancers were removed. The top 10 elements in each data source can be found in Table 1. 
Table 1. Distribution of the top 10 elements in each data source.

\begin{tabular}{|c|c|c|}
\hline \multicolumn{2}{|c|}{ Code and verbatim description } & \multirow[t]{2}{*}{ Record, n (\%) } \\
\hline Genes & & \\
\hline TP53 & tumor protein $\mathrm{p} 53$ & $553(54.70)$ \\
\hline$K R A S$ & KRAS proto-oncogene, GTPase & $292(28.88)$ \\
\hline$M L L 2$ & lysine methyltransferase $2 \mathrm{D}^{\mathrm{a}}$ & $173(17.11)$ \\
\hline$L R P 1 B$ & LDL receptor related protein $1 \mathrm{~B}$ & $171(16.91)$ \\
\hline$M L L 3$ & lysine methyltransferase $2 C^{a}$ & $150(14.84)$ \\
\hline$A P C$ & APC regulator of WNT signaling pathway & $141(13.95)$ \\
\hline$A R I D 1 B$ & AT-rich interaction domain 1B & $137(13.55)$ \\
\hline FAT1 & FAT atypical cadherin 1 & $134(13.25)$ \\
\hline$P R K D C$ & protein kinase, DNA-activated, catalytic subunit & $128(12.66)$ \\
\hline ARIDIA & AT-rich interaction domain $1 \mathrm{~A}$ & $126(12.46)$ \\
\hline \multicolumn{3}{|l|}{ Diagnosis $^{\mathbf{b}}$} \\
\hline Z02.9 & Work Status Exam (RTW) & $204(25.66)$ \\
\hline $\mathrm{I} 10$ & Hypertension (HTN) Chronic & $142(17.86)$ \\
\hline 401.9 & HYPERTENSION NOS & $138(17.36)$ \\
\hline 272.4 & HYPERLIPIDEMIA NEC/NOS & $116(14.59)$ \\
\hline R91.8 & Mass Lung & $113(14.21)$ \\
\hline V68.9 & ADMINISTRTVE ENCOUNT NOS & $106(13.33)$ \\
\hline $\mathrm{Z} 00.00$ & Maintenance Health (HM) & $101(12.70)$ \\
\hline E78.5 & Dyslipidemia NOS & $93(11.70)$ \\
\hline V72.83 & PREOP EXAMINATION NEC & $79(9.94)$ \\
\hline V70.0 & ROUTINE MEDICAL EXAM & $79(9.94)$ \\
\hline \multicolumn{3}{|l|}{ Lab tests ${ }^{\mathrm{c}}$} \\
\hline $777-3$ & Platelets [\#/volume] in Blood by Automated count & $991(99.40)$ \\
\hline $2160-0$ & Creatinine [Mass/volume] in Serum or Plasma & $988(99.10)$ \\
\hline 965763 & Hematocrit [Volume Fraction] of Blood by Automated count & $985(98.80)$ \\
\hline $718-7$ & Hemoglobin [Mass/volume] in Blood & $985(98.80)$ \\
\hline $788-0$ & Erythrocyte distribution width [Ratio] by Automated count & $985(98.80)$ \\
\hline $789-8$ & Erythrocytes [\#/volume] in Blood by Automated count & $985(98.80)$ \\
\hline 1749545 & Leukocytes [\#/volume] in Blood by Automated count & $985(98.80)$ \\
\hline $787-2$ & MCV [Entitic volume] by Automated count & $985(98.80)$ \\
\hline 337180 & Potassium [Moles/volume] in Serum or Plasma & $975(97.80)$ \\
\hline 383903 & Sodium [Moles/volume] in Serum or Plasma & $973(97.60)$ \\
\hline \multicolumn{3}{|c|}{ Family historical records ${ }^{b}$} \\
\hline V47.2 & Other cardiorespiratory problems & $205(29.54)$ \\
\hline 429.9 & Heart disease, unspecified & $205(29.54)$ \\
\hline 429.89 & Other ill-defined heart diseases & $205(29.54)$ \\
\hline 162.9 & Malignant neoplasm of bronchus and lung, unspecified & $133(19.16)$ \\
\hline 162.8 & Malignant neoplasm of other parts of bronchus or lung & $130(18.73)$ \\
\hline 272.4 & Other and unspecified hyperlipidemia & $124(17.87)$ \\
\hline 434.91 & Cerebral artery occlusion, unspecified with cerebral infarction & $104(14.99)$ \\
\hline
\end{tabular}




\begin{tabular}{|c|c|c|}
\hline \multicolumn{2}{|c|}{ Code and verbatim description } & \multirow{2}{*}{$\begin{array}{l}\text { Record, n (\%) } \\
84(12.10)\end{array}$} \\
\hline 799.9 & Other unknown and unspecified cause of morbidity and mortality & \\
\hline 311 & Depressive disorder, not elsewhere classified & $72(10.37)$ \\
\hline 447.9 & Unspecified disorders of arteries and arterioles & $63(9.08)$ \\
\hline \multicolumn{3}{|l|}{ Medication $^{\mathbf{d}}$} \\
\hline 5956 & Iohexol & $399(72.41)$ \\
\hline 1359867 & Sodium Chloride 9 MG/ML Prefilled Syringe & $374(67.88)$ \\
\hline 1807638 & 20 ML Sodium Chloride 9 MG/ML Injection & $304(55.17)$ \\
\hline 1807639 & 1000 ML Sodium Chloride 9 MG/ML Injection & $298(54.08)$ \\
\hline 1740467 & 2 ML Ondansetron $2 \mathrm{MG} / \mathrm{ML}$ Injection & $251(45.55)$ \\
\hline 4337 & Fentanyl & $224(40.65)$ \\
\hline 314659 & heparin sodium, porcine & $207(37.57)$ \\
\hline 847630 & $\begin{array}{l}\text { Calcium Chloride } 0.0014 \text { MEQ/ML / Potassium Chloride } 0.004 \text { MEQ/ML / Sodium } \\
\text { Chloride } 0.103 \mathrm{MEQ} / \mathrm{ML} \text { / Sodium Lactate } 0.028 \mathrm{MEQ/ML} \mathrm{Injectable} \mathrm{Solution}\end{array}$ & $202(36.66)$ \\
\hline 198440 & Acetaminophen 500 MG Oral Tablet & $188(34.12)$ \\
\hline 1808234 & 10 ML Propofol 10 MG/ML Injection & $163(29.58)$ \\
\hline \multicolumn{3}{|l|}{ Cancers $^{\mathbf{b}}$} \\
\hline 162.9 & Malignant neoplasm of bronchus and lung, unspecified & $231(22.85)$ \\
\hline 153.9 & Malignant neoplasm of colon, unspecified site & $124(12.27)$ \\
\hline 155 & Malignant neoplasm of liver, primary & $118(12.67)$ \\
\hline 157.9 & Malignant neoplasm of pancreas, part unspecified & $116(11.47)$ \\
\hline 183 & Malignant neoplasm of ovary & $85(8.41)$ \\
\hline 185 & Malignant neoplasm of prostate & $80(7.91)$ \\
\hline 171.9 & Malignant neoplasm of connective and other soft tissue, site unspecified & $68(6.73)$ \\
\hline 193 & Malignant neoplasm of thyroid gland & $55(5.44)$ \\
\hline 174.9 & Malignant neoplasm of breast (female), unspecified & $53(5.24)$ \\
\hline - $^{\mathrm{e}}$ & - & - \\
\hline
\end{tabular}

${ }^{\mathrm{a} C}$ Current standard gene symbols: $M L L 2$ is now $K M T 2 D ; M L L 3$ is now KMT2C.

${ }^{\mathrm{b}}$ International Statistical Classification of Diseases (ninth revision) code and description.

${ }^{\mathrm{c}}$ LOINC code and description.

${ }^{\mathrm{d}} \mathrm{RxN}$ Norm code and description.

${ }^{\mathrm{e}} \mathrm{A}$ tenth item is not included.

\section{Data Preprocessing and Data Modeling Based on FHIR and RDF}

We adapted FHIR-based data models from our previous work [37] employing FHIR resources to represent data elements of genetic reports and structured electronic health record data for phenome-wide association studies. Specifically, we represented genetic entries with the existing profile Observation-genetics, extended from the resource Observation. The lab test, diagnosis, and medication entries were represented with the resources Observation, Condition, and Medication, respectively, and were identified by encounters (eg, billing and electronic health record encounters) and service date. The family historical records entities were represented with the resource FamilyMemberHistory as diseases and were encoded with the attributed condition. All the resources were associated with the resource Patient. We further converted the JavaScript object notation-formatted FHIR data to RDF format based on the conversion rules, where (1) all the string-type values were considered as the entities in the RDF graph, and (2) all the values of the resources were considered as the object of the data-type property - named after the resource for the subject resource Patient. We illustrated an example of data representation based on FHIR and RDF in Figure 2. 
Figure 2. An example of data representation based on Fast Healthcare Interoperability Resources (FHIR) and Resource Description Framework (RDF): 2 JavaScript object notation-formatted FHIR representations for patients 1 and 2 are merged and converted into 1 RDF graph.
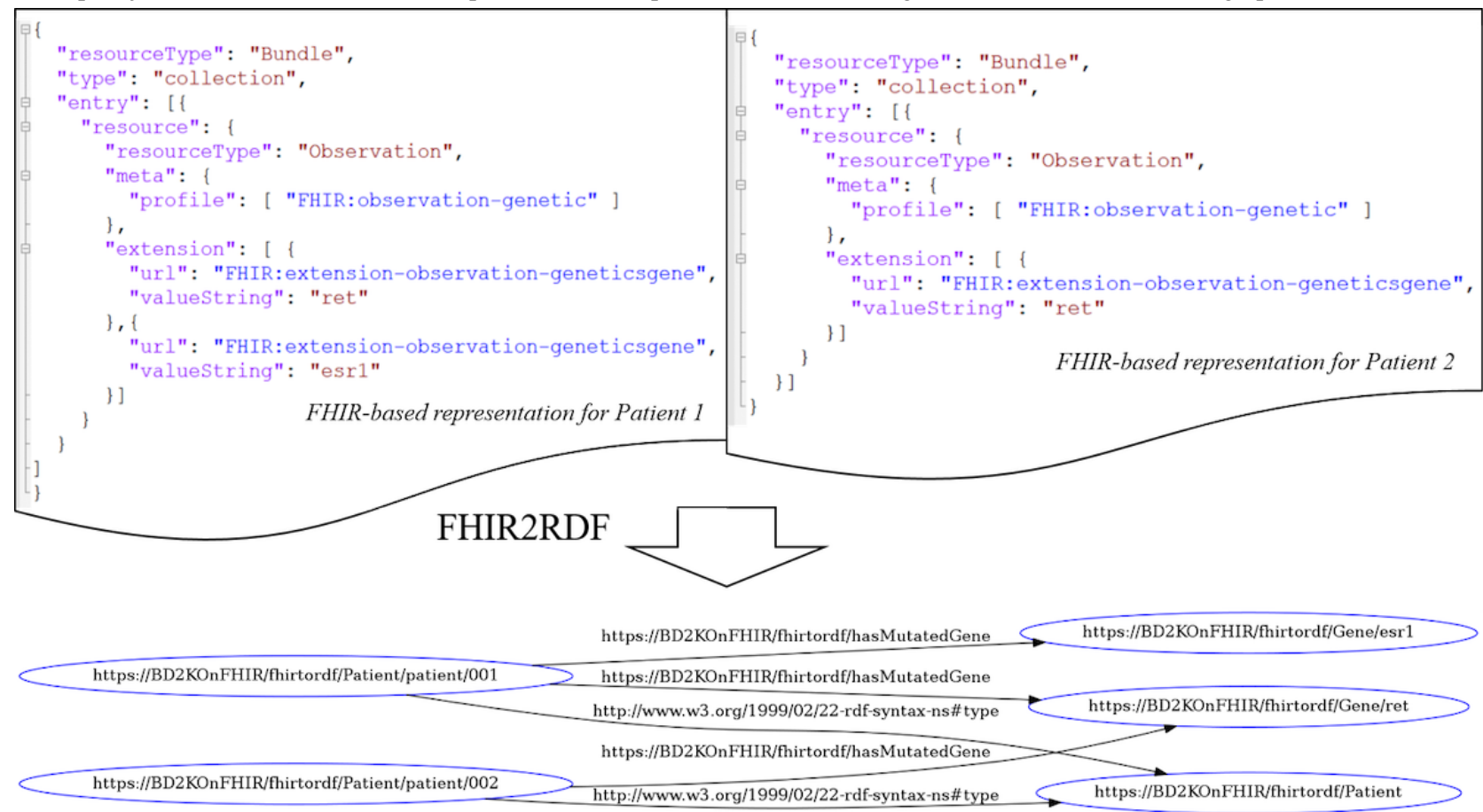

\section{Feature Generation and Cancer Prediction}

\section{Bag of Features}

Bag of features is analogous to the bag-of-words representation and characterizes a sample with an orderless collection of features [38]. In this study, we used bag of features based on the attribute values from the FHIR model. Specifically, categorical values of mutated genes, lab test results, disease diagnoses, medications for treatment, and historical family disease diagnoses were collected as the features from Observation-genetics, Observation, Condition, Medication, and FamilyMemberHistory, respectively. Additionally, patient demographic features, such as age and gender, were also used.

\section{Topological Features}

In order to train a model with the features generated from the input RDF data, we adapted a methodology [21] that considered RDF graph as a network, $G(V, E)$ with a set of vertices $V$ and a set of edges $E$, where $V$ has 7 types of vertices (ie, genetics, lab tests, diagnosis, medication, family historical records, demographics, and patients) and $E$ represents associations between the 6 types of vertices (ie, genetics, lab tests, diagnosis, medication, family historical records, demographics) and patients. We used the graph embedding method to learn the features of the patients, where a patient could be represented by a vector embedded within the topological structure of the patient in the network $G$. Node2vec [30] is a state-of-art graph embedding method that vectorizes the vertices of a network based on the topology of the network by maximizing the probability of observing the neighborhood $N(u)$ of each node $u$ in $G$ :

$$
\max \sum_{u \in V}\left[-\log Z_{u}+\sum_{v \in N(u)} f(v) \cdot f(u)\right],
$$

where

$$
Z_{u}=\sum_{v \in V} \exp (f(v) \cdot f(u))
$$

and $f(\cdot)$ was the feature representation of a node. In addition, we also generated a $|V| \times|V|$ adjacency matrix from $G$, where each cell of the matrix was set to 1 if there was a connection between nodes, otherwise the cell was set to 0 .

We modeled cancer prediction as a multiple-label classification problem, where a given patient was represented with $\mathrm{k}$-dimensional features, and a model categorized the patient into precisely 1 of 9 cancer types: colon cancer (ICD-9: 153.9), pancreas cancer (ICD-9: 157.9), ovary cancer (ICD-9: 183), prostate cancer (ICD-9: 185), connective and other soft tissue cancer (ICD-9: 171.9), thyroid gland cancer (ICD-9: 193), breast cancer (ICD-9: 174.9), liver cancer (ICD-9: 155), and bronchus and lung cancer (ICD-9: 162.9).

\section{Experiment Design}

\section{Overview}

There were 2 main drivers of this study: (1) from a methodological perspective- how could generated features be coordinated with classification methods in a favorable manner to achieve satisfactory prediction?-and (2) from a data perspective - which data sources, especially genetic data, are preferable in prediction? Our experiment was thus conducted as a sequence of 6 distinct tasks.

\section{Task 1: Comparison of Combinations of Features and Popular Classification Methods}

A comparison of 3 feature generation methods - bag of features, Node2vec, and bag of features+Node2vec (ie, a linear combination of bag of features and Node2vec) - was conducted. Seven classification methods - random forest [39], naive Bayes [40], logistic regression [41], support vector machine [42], deep 
neural network [43], convolutional neural network [44], and graph convolutional networks [45]—were used.

\section{Task 2: Comparison of Combinations of Data Sources}

There were 5 types of data sources used in this study. We took all possible combinations of the data sources into consideration and studied how the features generated from these sources affected the results.

\section{Task 3: Comparison of Predictions for Each Cancer}

To understand how the prediction varied in different cancers predictions, we conducted 9 prediction tasks for all the cancers to study.

\section{Task 4: Analysis of Feature Contribution for Each Cancer Prediction}

To interpret the model and understand which features were important to each cancer, we studied the features that contributed most to the prediction of cancer.

\section{Task 5: Time Effect of Cancer Prediction}

To understand how the prediction could be made precisely prior to a certain amount of time of the diagnosis, we studied the prediction based on data collected at different duration, ranging from 0 to 24 months, in advance.

\section{Task 6: Prediction of Cancer of Unknown Primary Patients}

We identified the 43 primary cancers from 81 patients with cancer of unknown primary based on the diagnosis records to understand how the proposed method performed for real cancer predictions. Please note, no patients with pancreas cancer of unknown primary were identified, and therefore, pancreatic cancer was not considered in this task.

\section{Feature Selection and Classification}

Two methods were used to generate features: bag of features and Node2vec. For bag of features, all genes, diseases, drugs in genetics, diagnosis, medication, and family historical records were considered as features. For the lab tests, the values were converted into categorical values (Null, Normal, or Abnormal) based on the normal range defined in the unified data platform. To avoid overfitting, the features were reduced to $d=\{10,20,30,40,50,60,70,80,90,100\}$ based on information gain [46]. For Node2vec, the parameter ranges for the grid search were specified as the number of walks $\gamma=\{10,40\}$, return $P=\{0.5,1.0,2.0\}, \quad$ in-out $q=\{0.5,1.0,2.0\}, \quad$ dimension $d=\{10,20,30,40,50,60,70,80,90,100\}$, window size $w=\{5,10\}$, and walk length $t=\{40,80\}$.

Four popular machine learning models and 3 deep learning models were used for classification. For machine learning methods, the following settings were used: L2 regularization for logistic regression, type C-SVC and linear kernel for support vector machine, 500 trees for random forest, and default settings for naive Bayes. For deep learning methods, the following structure were used: 5 dense layers with dimensions $\{256$, $256,128,64,10\}$ (4 rectified linear unit [ReLU] activation functions with 0.5 dropout rate and 1 softmax activation function) for deep neural network, 3 convolution layers with filters $\{256,256,256\}$ (3 ReLU activation functions and maxpooling layers with 0.5 dropout rate) followed with 4 dense layers with dimensions $\{256,128,64,10\}$ (3 ReLU activation functions with 0.5 dropout rate and 1 softmax activation function) for convolutional neural network, and 2 graph convolutional layers with channels $\{64,10\}$ (1 ReLU activation function with 0.5 dropout rate and 1 softmax activation function) for graph convolutional networks.

Node2vec was obtained from the Node2vec library [47]. The logistic regression classifier was obtained from the LIBLINEAR library [48]; naive Bayes, random forest, and information gain algorithms were obtained from Weka library [49], support vector machine was obtained from LIBSVM [50]. Deep neural network and graph convolutional networks were constructed based on Keras library [51]. Graph convolutional networks algorithms were obtained from Spektral library [52].

\section{Validation and Evaluation Metrics}

We used conventional 10-fold cross-validation for the evaluation, where 10 independent iterations of training and testing were conducted, and a random partition of the original samples into 10 equal-size subsamples was performed. To assess the quality of classification, we used area under the receiver operating characteristic curve (AUROC) [53]. In addition, the area under the precision-recall curve (AUPRC) [53] was used as a supplementary metric characterizing the results for imbalanced classes [54,55]. AUROC and AUPRC scores were calculated using the Java Receiver Operating Characteristic library [56] and Weka evaluation package [57].

\section{Results}

\section{Combinations of Features and Popular Classification Methods}

Table 2 shows the best performance result was achieved by using bag of features+Node2vec and random forest (AUROC 96.19\%) (AUPRC: Table S1, Multimedia Appendix 1). Generally, using bag of features+Node2vec outperformed using bag of features $(+1.27 \%)$ and Node2vec $(+1.41 \%)$. Although we observed that machine learning-based methods outperformed deep learning-based methods, in general, the best deep learning-based approach (AUROC 95.12\%) was second to the best machine learning-based approach by only 1 percentage-point difference (outperforming the remaining machine learning-based approaches). As our implementation of deep learning models is based on simple architectures, the deep learning models with more complex architectures have the potential to facilitate feature generation and may directly contribute to improvements in cancer prediction. 
Table 2. Prediction performance (area under the receiver operatic characteristic curve) for combinations of features and classification methods.

\begin{tabular}{llll}
\hline Classifiers & \multicolumn{2}{l}{ Feature generation algorithm } & \\
& Bag of features & Node2vec & Bag of features+Node2vec \\
& AUROC $^{\mathrm{a}}(\%)$ & AUROC $(\%)$ & AUROC (\%) \\
\hline Random forest & 94.82 & 91.89 & 96.19 \\
Naive Bayes & 92.30 & 92.91 & 94.76 \\
Logistic regression & 86.68 & 85.25 & 89.39 \\
Support vector machine & 84.62 & 83.92 & 86.72 \\
Convolutional neural network & 64.14 & 63.36 & 57.68 \\
Deep neural network & 92.56 & 92.87 & 95.12 \\
Graph convolutional networks & 79.67 & 83.62 & 83.83 \\
\hline
\end{tabular}

aAUROC: area under the receiver operating characteristic curve.

\section{Combinations of Data Sources}

Table 3 shows better results were achieved by the model DML+G (diagnosis, medication, lab test, and genetic information; AUROC 96.56\%). Steady improvement is obtained when more features are used (AUPRC: Table S2, Multimedia Appendix 1). For example, increasing average AUROCs $(75.49 \%, 82.65 \%, 87.98 \%$, and $91.74 \%)$ are achieved by adding 1 to 5 features successively without using genetic information. Table 3 also presents the importance of the features, where lab test is the most important feature (91.00\%), followed by diagnosis $(73.12 \%)$, medication $(72.83 \%)$, and family historical records $(65.01 \%)$. We also demonstrated the value of genetic information for cancer prediction - an average improvement of $10.52 \%$ was reached. Interestingly, such improvement is weakened when more feature types are used $(+15.76 \%$ for using 1 feature type, $+10.45 \%$ for 2 feature types, $+6.92 \%$ for 3 feature types, and $+4.45 \%$ for 4 feature types). Table 3 also indicates the potential of using diverse types of features alternatively when genetic information is not available. 
Table 3. Prediction performance for combinations of data sourcing with bag of features+Node $2 \mathrm{vec}$ and random forest algorithms.

\begin{tabular}{|c|c|c|}
\hline \multirow[t]{2}{*}{ Feature types } & \multicolumn{2}{|l|}{ AUROC $^{\mathrm{a}}(\%)$} \\
\hline & Base feature set & With genetic information \\
\hline \multicolumn{3}{|l|}{1 feature type } \\
\hline $\mathrm{G}^{\mathrm{b}}$ & 73.12 & 90.89 \\
\hline$D^{c}$ & 65.01 & 88.37 \\
\hline $\mathrm{H}^{\mathrm{d}}$ & 91.00 & 95.80 \\
\hline $\mathrm{L}^{\mathrm{e}}$ & 72.83 & 89.94 \\
\hline$M^{f}$ & 73.21 & 90.92 \\
\hline \multicolumn{3}{|l|}{2 feature types } \\
\hline DH & 91.55 & 96.09 \\
\hline DL & 77.09 & 90.88 \\
\hline DM & 91.30 & 95.92 \\
\hline HL & 71.53 & 89.02 \\
\hline MH & 91.22 & 95.75 \\
\hline ML & 91.98 & 96.01 \\
\hline \multicolumn{3}{|l|}{3 feature types } \\
\hline DHL & 76.76 & 91.28 \\
\hline DMH & 91.76 & 96.56 \\
\hline DML & 91.43 & 95.76 \\
\hline MHL & 91.74 & 96.19 \\
\hline \multicolumn{3}{|l|}{4 feature types } \\
\hline DMHL & 73.12 & 90.89 \\
\hline
\end{tabular}

${ }^{a}$ AUROC: area under the receiver operating characteristic curve.

${ }^{\mathrm{b}} \mathrm{G}$ : genetic information.

${ }^{\mathrm{c}} \mathrm{D}$ : diagnosis.

${ }^{\mathrm{d}} \mathrm{H}$ : family historical records.

${ }^{\mathrm{e}} \mathrm{L}$ : lab test.

${ }^{\mathrm{f}} \mathrm{M}$ : medication.

\section{Predictions for Each Cancer}

Table 4 shows that the proposed method achieved high AUROC values across all 9 cancer types (AUPRC: Table S3, Multimedia Appendix 1), especially for thyroid gland (AUROC 99.80\%), prostate $(99.76 \%)$, breast $(98.53 \%)$, ovary $(98.29 \%)$, connective

and other soft tissue (96.05\%), and liver $(95.41 \%)$. Genetic information improved the predictions in general $(P<.001)$ based on a Wilcoxon signed-rank test [58], specifically for thyroid gland cancer $(P=.03)$, ovary cancer $(P=.03)$, connective and other soft tissue cancer $(P=.03)$, liver cancer $(P=.03)$, and colon cancer $(P=.03)$. 
Table 4. Prediction performance for 9 cancer types.

\begin{tabular}{|c|c|c|}
\hline \multirow[t]{2}{*}{ Cancer (ICD-9 $9^{\mathrm{a}}$ code) } & \multicolumn{2}{|c|}{$\operatorname{AUROC}^{\mathrm{b}}(\%)$} \\
\hline & $\mathrm{DML}^{\mathrm{c}}$ & $\mathrm{DML}+\mathrm{G}^{\mathrm{d}}$ \\
\hline Malignant neoplasm of thyroid gland (193) & 99.55 & 99.80 \\
\hline Malignant neoplasm of prostate (185) & 98.43 & 99.76 \\
\hline Malignant neoplasm of breast (female), unspecified (174.9) & 96.80 & 98.53 \\
\hline Malignant neoplasm of ovary (183) & 95.73 & 98.29 \\
\hline Malignant neoplasm of connective and other soft tissue, site unspecified (171.9) & 82.39 & 96.05 \\
\hline Malignant neoplasm of liver, primary (155) & 91.39 & 95.41 \\
\hline Malignant neoplasm of pancreas, part unspecified (157.9) & 91.07 & 95.41 \\
\hline Malignant neoplasm of bronchus and lung, unspecified (162.9) & 90.61 & 93.24 \\
\hline Malignant neoplasm of colon, unspecified site (153.9) & 79.88 & 92.56 \\
\hline
\end{tabular}

${ }^{a}$ ICD-9: International Statistical Classification of Diseases, ninth revision.

${ }^{b}$ AUROC: area under the receiver operating characteristic curve.

${ }^{\mathrm{c}}$ DML: diagnosis, medication, and lab test.

${ }^{\mathrm{d}} \mathrm{DML}+\mathrm{G}$ : diagnosis, medication, and lab test, and genetic information.

\section{Feature Contributions for Each Cancer Prediction}

Our analysis examines the feature contribution based on SHAP values [59] for the cancer prediction and selects the top 5 features interpretable for each cancer (Figure 3). Frequent common features are lab tests (11/17); cancer antigen 19-9 in serum or plasma (2.03\%), carbohydrate antigen 19-9, S (1.76\%), and cancer antigen 125 in serum or plasma by immunoassay $(2.59 \%)$ are the most common features across all the cancer types. These lab tests are considered to be predictive biomarkers for prognosis and chemotherapeutic effect for carcinomas [60-63]. Two genes-KRAS proto-oncogene, GTPase homolog $(K R A S)(1.46 \%)$ and adenoma polyposis coli regulator of WNT signaling pathway $(A P C)(1.60 \%)$ contribute the most cancer predictions. KRAS is the most commonly mutated oncogene in human cancers. The sustained expression and signaling of KRAS results in the progress of many cancers thus make it the high-priority target in clinical therapeutic implications [64]. $A P C$ participates in a cytoplasmic complex and its mutation triggers negatively regulating canonical WNT signaling. APC counteracts proliferation, facilitates apoptosis, and suppresses tumor progression, thus APC-deficient tumors drive colorectal and gastric cancers $[65,66]$.

Lab tests testosterone $(2.49 \%)$ and prostate-specific antigen in serum or plasma $(2.29 \%)$ were found to be the major contributors to prostate cancer prediction. Evidence supports the androgen hypothesis, where prostate cancer development and progression are related to androgens. These findings drive the studies to explore the correlation between testosterone and prostate cancer development and progression [67,68]. For thyroid gland cancer prediction, thyroglobulin antibody in serum or plasma by immunoassay $(2.69 \%)$, thyroglobulin in serum or plasma (0.58\%), T4 (thyroxine) (0.62\%), and gene telomerase reverse transcriptase (TERT) (SHAP value $0.59 \%$ ) were found to be the major contributors. Associations between autoimmune thyroiditis and thyroid cancer have been documented [69] in studies where thyroid autoimmunity was assessed by measuring thyroglobulin antibody and thyroid peroxidase antibody [70,71]. Thyroglobulin in serum also plays a key role in the surveillance of differentiated patients with thyroid cancer [72]. TERT promoter mutations have been found to be strongly associated with different pathological types of thyroid cancers and are considered as the biomarker to the preoperative diagnosis and prognosis of thyroid cancers [73]. Cancer antigen 15-3 in serum or plasma (1.57\%) and cancer antigen 15-3 (CA 15-3) S (0.98\%) lab tests are the major contributors to breast cancer prediction. Cancer antigen 15-3 is a protein made by a variety of cells, particularly breast cancer cells, and the cancer antigen 15-3 test is A biomarker test used to monitor breast cancer [74]. In addition, the cancer markers alpha-fetoprotein, tumor marker, $\mathrm{S}(0.78 \%)$ and epidermal growth factor receptor (EGFR) (1.56) were found to be the main contributors for the prediction of cancers of the liver [75] and bronchus and lung [76]. In our study, sex appears to be the major contributor to prediction of cancers of the breast $(0.76 \%)$, prostate $(0.92 \%)$, and ovary $(1.16 \%)$. 
Figure 3. Top 5 features contributing to cancer prediction.

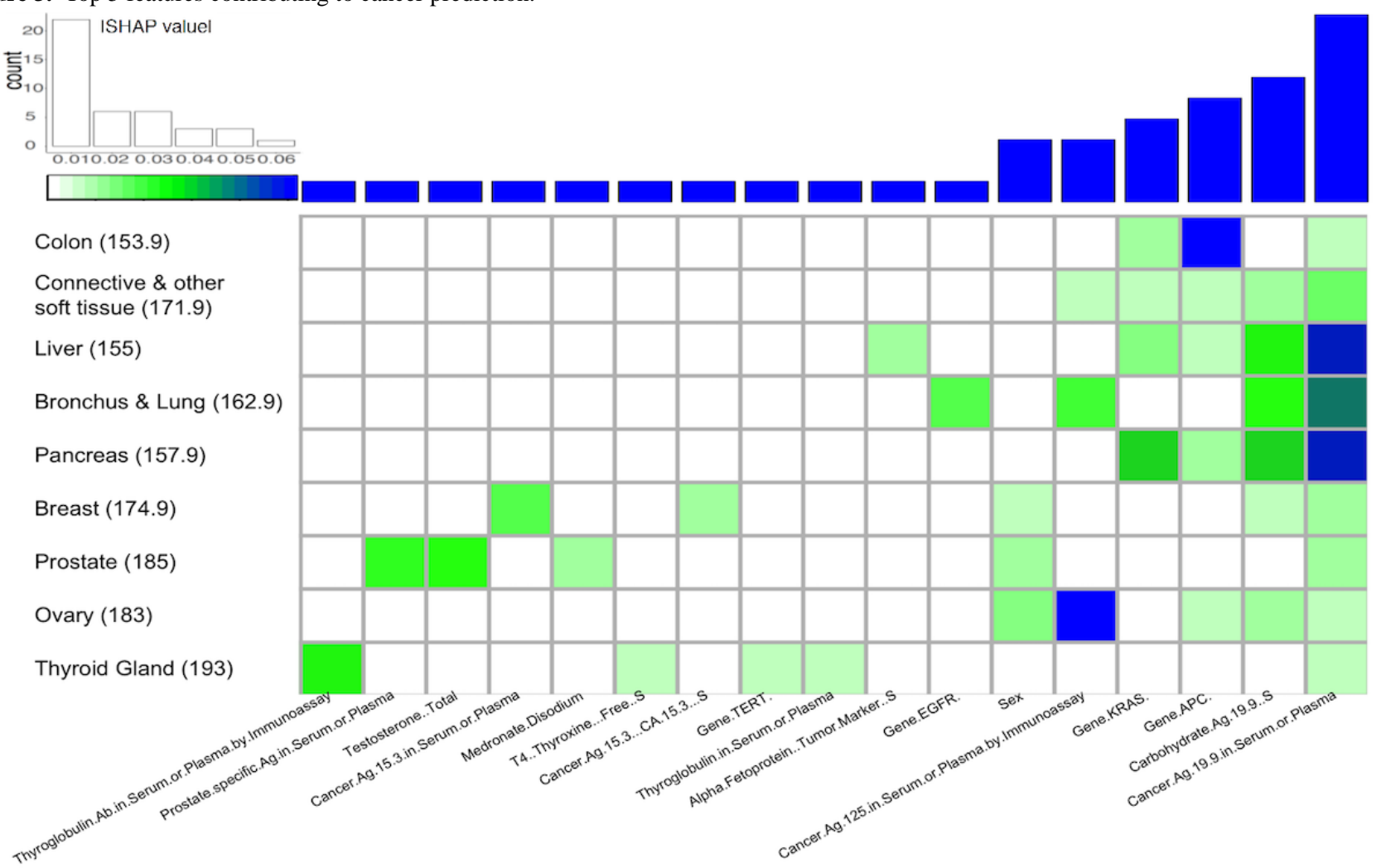

\section{Time Effect of Cancer Prediction}

Table 5 shows predictions based on different resources with different combinations of time-dependent (diagnosis, medication, and lab test) features (AUPRC: Table S4, Multimedia Appendix 1). Among the 7 models, diagnosis and lab test were the best (average AUROC 90.31\%). In general, the performance of prediction decreases as more time increases prior to the formal diagnosis. For example, the average performance was reduced from $92.37 \%$ to $77.18 \%$ from 0 months to 24 months in advance, with an average decrease of $3.04 \%$. Table 5 also demonstrates the performance of the model (ie, diagnosis, medication, lab test, and genetic information) based on genetic information (AUROC $91.38 \%$ at 24 months in advance, an improvement of $+11.38 \%$ over diagnosis, medication, and lab test). The difference between the two increase as time increases (eg, 1.06 for 0 months to $11.38 \%$ for 24 months), which suggests the importance of genetic testing at early stages.

Table 5. Prediction performance (AUROC) 0 months to 24 months in advance.

\begin{tabular}{|c|c|c|c|c|c|c|c|c|}
\hline \multirow[t]{3}{*}{ Months } & \multicolumn{8}{|l|}{ Feature type } \\
\hline & $\mathrm{DML}+\mathrm{G}^{\mathrm{a}}$ & $\begin{array}{l}\text { Diagnosis, } \\
\text { medication, } \\
\text { and lab test }\end{array}$ & $\begin{array}{l}\text { Diagnosis and } \\
\text { lab test }\end{array}$ & $\begin{array}{l}\text { Diagnosis and } \\
\text { medication }\end{array}$ & $\begin{array}{l}\text { Medication } \\
\text { and lab test }\end{array}$ & Diagnosis & Medication & Lab test \\
\hline & $\operatorname{AUROC}^{\mathrm{b}}(\%)$ & AUROC (\%) & AUROC (\%) & AUROC (\%) & AUROC $(\%)$ & AUROC (\%) & AUROC (\%) & AUROC $(\%)$ \\
\hline 0 & 99.43 & 98.36 & 98.41 & 97.89 & 88.67 & 97.90 & 70.39 & 87.93 \\
\hline 1 & 98.08 & 95.62 & 95.51 & 94.31 & 86.83 & 94.53 & 71.01 & 86.67 \\
\hline 3 & 96.52 & 93.16 & 93.22 & 90.74 & 84.85 & 91.20 & 69.36 & 84.18 \\
\hline 6 & 95.21 & 89.69 & 89.91 & 85.53 & 83.09 & 85.26 & 68.12 & 83.38 \\
\hline 12 & 93.17 & 84.39 & 84.60 & 78.20 & 80.56 & 78.21 & 66.76 & 79.99 \\
\hline 24 & 91.38 & 80.01 & 80.20 & 71.81 & 77.73 & 71.71 & 66.22 & 78.35 \\
\hline
\end{tabular}

${ }^{\mathrm{a} D M L}+\mathrm{G}$ : diagnosis, medication, and lab test, and genetic information.

b AUROC: area under the receiver operating characteristic curve. 


\section{Prediction of Patients With Cancer of Unknown Primary}

In spite of the challenge in identifying patients with cancer of unknown primary in the clinical setting, hybrid features-the diagnosis, medication, lab test, and genetic information model—outperformed the diagnosis, medication, and lab test model (AUPRC: Table S5, Multimedia Appendix 1), and bag of features+Node2vec outperform the bag of features and Node2vec in most cases. Table 6 shows promising prediction results for 4 cancers, especially for breast (AUROC 92.31\%), connective and other soft tissue (AUROC 92.31\%). Cancers of the liver and lung have the largest number of patients (24/43) and also achieved satisfactory predictions (AUROCs $88.21 \%$ and $85.51 \%$ ). We also note that the proposed method performed suboptimally in predicting cancer of the colon (AUROC $52.56 \%$ ). Prediction of the prostate, thyroid gland, and colon cancers had better results for the bag of features+Node2vec model with diagnosis, medication, and lab test features and for the bag of features or Node2vec model with diagnosis, medication, lab test, and genetic information features (Table S6, Multimedia Appendix 1), suggesting a more flexible strategy of model adaptation for the prediction of cancer of unknown primary in practice.

Table 6. AUROC (\%) of prediction for 9 cancer types.

\begin{tabular}{|c|c|c|c|}
\hline \multirow[t]{2}{*}{ Cancer (ICD-9 $9^{\mathrm{a}}$ code) } & \multicolumn{2}{|c|}{$\operatorname{AUROC}^{\mathrm{b}}(\%)$} & \multirow[t]{2}{*}{ Patients, $\mathrm{n}$} \\
\hline & $\mathrm{DML}^{\mathrm{c}}$ & $\mathrm{DML}+\mathrm{G}^{\mathrm{d}}$ & \\
\hline Malignant neoplasm of breast (female), unspecified (174.9) & 83.97 & 92.31 & 4 \\
\hline Malignant neoplasm of connective and other soft tissue, site unspecified (171.9) & 53.21 & 92.31 & 4 \\
\hline Malignant neoplasm of liver, primary (155) & 84.10 & 88.21 & 13 \\
\hline Malignant neoplasm of bronchus and lung, unspecified (162.9) & 74.43 & 85.51 & 11 \\
\hline Malignant neoplasm of ovary (183) & 65.85 & 80.49 & 2 \\
\hline Malignant neoplasm of prostate (185) & 91.67 & 79.17 & 3 \\
\hline Malignant neoplasm of thyroid gland (193) & 90.24 & 75.61 & 2 \\
\hline Malignant neoplasm of colon, unspecified site (153.9) & 64.74 & 52.56 & 4 \\
\hline
\end{tabular}

${ }^{\text {a } I C D-9: ~ I n t e r n a t i o n a l ~ S t a t i s t i c a l ~ C l a s s i f i c a t i o n ~ o f ~ D i s e a s e s, ~ n i n t h ~ r e v i s i o n . ~}$

${ }^{b}$ AUROC: area under the receiver operating characteristic curve.

${ }^{\mathrm{c} D M L}$ : diagnosis, medication, and lab test.

${ }^{\mathrm{d}} \mathrm{DML}+\mathrm{G}$ : diagnosis, medication, and lab test, and genetic information.

\section{Discussion}

It is recognized that both genetic and nongenetic factors may lead to the development of cancers, and they are, therefore, considered to be risk factors in the plethora of cancer prediction models based on statistical analysis; this leads to performance (eg, AUROC) ranging from $60 \%$ to $90 \%$ [77]. For example, the variables of high DNA load of high-risk human papillomavirus, age, marital status, smoking status, and age at sexual debut are the critical factors to achieve the AUROC $90 \%$ in the prediction of cervical intraepithelial neoplasia grade 2 or worse [78]. DNA methylation-based markers-based method achieves AUROC 93\% in the detection of preinvasive neoplasia and cervical cancer [79]. Computational methods (eg, machine learning and deep learning) have been adapted to provide solutions for cancer prediction challenges in a controlled environment (eg, UCI machine repository [80]). For example, linear support vector machines achieved AUROC 96.7\% [81] and k-nearest neighbors classifier achieved an accuracy of $99.28 \%$ [82] for breast cancer prediction.

Public genetic expression databases (eg, The Cancer Genome Atlas) are frequently used to train diverse deep learning models. A convolutional neural network-based model achieved accuracies of $93.9 \%$ to $95.0 \%$ in the prediction of 34 cancer types [83]. For lung, stomach, and breast cancer, AUROCs
$99.5 \%, 97.1 \%$, and $95.0 \%$, respectively, were achieved by a stacked sparse auto-encoder-based classification model [84]. Prostate cancer prediction achieved an AUROC of $95.5 \%$ with a genetic algorithm-optimized artificial neural network [85]. Accuracies of $95.3 \%$ for breast cancer, $57.9 \%$ for leukemia, and $84.9 \%$ for colon cancer were achieved by sample expansion based 1D convolutional neural network [86].

Electronic health records are utilized in cancer prediction. DeepPatient has proposed a novel unsupervised feature learning method based on autoencoders for disease prediction [87]. The overall AUROC was $77.3 \%$, where AUROCs of $88.7 \%$ for cancer of rectum and anus, $88.6 \%$ for cancer of the liver and intrahepatic bile duct, $85.9 \%$ for cancer of the prostate were predicted with a time interval of 12 months. Multiple studies have utilized electronic health record data to predict specific cancers, where AUROCs of $88.1 \%$ for lung cancer [88], 64.8\% for breast cancer [89], 85\% for pancreatic cancer [90] were achieved, and $85.7 \%$ precision and $60.0 \%$ recall were achieved for colorectal cancer [91]. Our method achieved AUROC $96.56 \%$ in general and outperformed the state-of-the-art methods for most cancer types. Specifically, prostate cancer $(99.8 \%)$, breast cancer (AUROC 98.5\%), liver cancer (95.4\%), and pancreas cancer (95.4\%) predictions results were better for our method. 
In this study, we designed and developed a network-based framework leveraging the FHIR resources and RDF for cancer prediction. Our contributions can be summarized as exploration of utilizing FHIR and RDF technology to provide a network-based representation for the prediction of patient health status, demonstrating the value of integrating the phenotypic and genetic features data sources to improve the accuracy and interpretability in cancer prediction models. To enable the standard representation of data, a FHIR-based representation was used as the core to support the network population and feature generation. It is one of the most popular clinical data standards and is widely used among modern electronic health record vendors and data providers, enabling the plug and play functionality of the proposed method to be used across the different institutions, and it provides the specification and tools to seamlessly convert to RDF format and support the efficient data communication based on the popular data exchanging formats, such as XML or JavaScript object notation.

This study demonstrated a solution for the prediction of unknown cancer in clinical practice. Despite the value of this work, there are several limitations that should be addressed.

First, the genetic alterations in the genetic reports provided in Foundation Medicine are all somatic mutations in tumors and are collected from somatic tissues. Thus, we could not differentiate the germline and somatic mutations in our model. The bias introduced to the system caused by a failure in capturing this difference weakens the findings of our study.

Second, as most genetic tests are based on specimens collected from the biopsy or surgery, the best-performing (diagnosis, medication, lab test, and genetic information) model introduced in Task 5 might not be adaptable as some medical organizations have limited access to genetic information available for study. We, therefore, consider that it is more practical to learn a large amount of phenotypical information for cancer prediction with the full utilization of existing generic information. On the other hand, as the costs of genetic testing are reduced, we believe that the genetic information will be increasingly used in prediction models for different tasks, which makes the proposed method a good reference as a pilot study.

Third, within 81 patients who have been documented as having cancer of unknown primary (from genetic reports), we could identify specific cancer types for 43 patients based on the review of patients' diagnostic report for task 6 . We understand that the limited data set used might affect result analysis, which is a limitation of this experiment. We also noticed that the proposed method performs differently in task 6, especially with some notable failures. Such failures indicate the patterns of the value distribution for the features learned in the training data are not the same as the patterns in the cancer of unknown primary. The cancer of unknown primary source is not considered a single type of cancer and is known to spread at the early stage without causing phenotypical symptoms at the origin site [92]. As such, the proposed model is affected in Task 5 accordingly.

Fourth, our experiment demonstrates the performance of the proposed method based on data collected over a varying timeline. Data were used in isolation to train classification models, ignoring the continuous changing of the measurable values of phenotypes (eg, lab tests) during cancer progression. The introduction of deep learning models, such as recurring neural networks [93] and long short-term memory [94], which are capable of processing time-series data may potentially improve predictions.

Fifth, cancers related to the same genetic alteration (eg, both colorectal and gastric cancers are related to the $A P C$ gene) inspire us to explore the potential of considering dependent phenotypes of the genetic alteration. With the utilization of phenotype and genotype dependence based on the ontology structure, a more sophisticated method can be designed to empower the prediction. In the future, we plan to reach out to other institutions to apply our method both with and without genetic information on diverse electronic health record systems. We consider it is necessary to adopt other medical data standards, such as Observational Health Data Sciences and Informatics Common Data Model [95], to cover the diversity. We are aware that there are some challenging issues in genetic data modeling with relational databases, such as how to anonymize and aggregate genomic data. We believe that the research community will develop solutions for handling these challenging issues. We will incorporate such developments into our framework as part of future work to better support these requirements. The data process and cancer prediction tools of this study are publicly available [96].

\section{Acknowledgments}

This work was supported by funding from Genentech Research Fund in Individualized Medicine, National Institute of Health, National Institute of General Medical Sciences (K99GM135488), Big Data to Knowledge (U01HG009450), and FHIRCat (R56EB028101).

\section{Conflicts of Interest}

None declared.

\section{Multimedia Appendix 1}

Supplementary tables.

[XLSX File (Microsoft Excel File), 16 KB-Multimedia Appendix 1]

\section{References}


1. Cancer. World Health Organization. 2018. URL: https://www.who.int/news-room/fact-sheets/detail/cancer [accessed 2021-05-11]

2. Islami F, Miller KD, Jemal A. Cancer burden in the United States—a review. Ann Cancer Epidemiol 2018;1:1-1. [doi: 10.21037/ace.2018.08.02]

3. Leading causes of death. Centers for Disease Control and Prevention. 2017. URL: https://www.cdc.gov/nchs/fastats/ leading-causes-of-death.htm [accessed 2021-05-11]

4. Cancer statistics. National Cancer Institute. 2020. URL: https://www.cancer.gov/about-cancer/understanding/statistics [accessed 2021-05-11]

5. Clarke M, Hass A. Cancer stem cells. In: Meyers RA, editor. Reviews in Cell Biology and Molecular Medicine. Weinheim: Wiley - VCH Verlag GmbH \& Co KGaA; 2004:221-241.

6. Blackadar CB. Historical review of the causes of cancer. World J Clin Oncol 2016 Feb 10;7(1):54-86 [FREE Full text] [doi: 10.5306/wjco.v7.i1.54] [Medline: 26862491]

7. Walsh M, Cadoo K, Salo-Mullen E, Dubard-Gault M, Stadler Z, Offit K. Genetic factors: hereditary cancer predisposition syndromes. In: Abeloff's Clinical Oncology. Amsterdam, Netherlands: Elsevier; 2020:180-208.

8. Garber JE, Offit K. Hereditary cancer predisposition syndromes. J Clin Oncol 2005 Jan 10;23(2):276-292. [doi: 10.1200/JCO.2005.10.042] [Medline: 15637391]

9. Nagy R, Sweet K, Eng C. Highly penetrant hereditary cancer syndromes. Oncogene 2004 Aug 23;23(38):6445-6470. [doi: 10.1038/sj.onc.1207714] [Medline: 15322516]

10. Friedman AA, Letai A, Fisher DE, Flaherty KT. Precision medicine for cancer with next-generation functional diagnostics. Nat Rev Cancer 2015 Dec;15(12):747-756 [FREE Full text] [doi: 10.1038/nrc4015] [Medline: 26536825]

11. Nath AS, Pal A, Mukhopadhyay S, Mondal KC. A survey on cancer prediction and detection with data analysis. Innovations Syst Softw Eng 2019 Aug 22;16(3-4):231-243. [doi: 10.1007/s11334-019-00350-6]

12. Cancer survival in England: national estimates for patients followed up to 2017. Office for National Statistics. URL: https:/ /www.ons.gov.uk/releases/cancersurvivalinenglandadultstageatdiagnosisandchildhoodpatientsfollowedupto2017 [accessed 2021-05-11]

13. Hawkes N. Cancer survival data emphasise importance of early diagnosis. BMJ 2019 Jan 25;364:1408. [doi: 10.1136/bmj.1408] [Medline: $\underline{30683652]}$

14. Tests for cancer of unknown primary. American Cancer Society. URL: https://www.cancer.org/cancer/ cancer-unknown-primary/detection-diagnosis-staging/how-diagnosed.html [accessed 2021-05-11]

15. Losa F, Soler G, Casado A, Estival A, Fernández I, Giménez S, Seguí. SEOM clinical guideline on unknown primary cancer (2017). Clin Transl Oncol 2018 Jan;20(1):89-96 [FREE Full text] [doi: 10.1007/s 12094-017-1807-y] [Medline: 29230692]

16. Cowie MR, Blomster JI, Curtis LH, Duclaux S, Ford I, Fritz F, et al. Electronic health records to facilitate clinical research. Clin Res Cardiol 2017 Jan;106(1):1-9 [FREE Full text] [doi: 10.1007/s00392-016-1025-6] [Medline: 27557678]

17. Denaxas SC, Morley KI. Big biomedical data and cardiovascular disease research: opportunities and challenges. Eur Heart J Qual Care Clin Outcomes 2015 Jul 01;1(1):9-16. [doi: 10.1093/ehjqcco/qcv005] [Medline: 29474568]

18. Jiang F, Jiang Y, Zhi H, Dong Y, Li H, Ma S, et al. Artificial intelligence in healthcare: past, present and future. Stroke Vasc Neurol 2017 Dec;2(4):230-243 [FREE Full text] [doi: 10.1136/svn-2017-000101] [Medline: 29507784]

19. Rajkomar A, Oren E, Chen K, Dai AM, Hajaj N, Hardt M, et al. Scalable and accurate deep learning with electronic health records. NPJ Digit Med 2018;1:18 [FREE Full text] [doi: 10.1038/s41746-018-0029-1] [Medline: $\underline{31304302]}$

20. Prince S. Computer Vision: Models, Learning, and Inference. Cambridge, UK: Cambridge University Press; 2012.

21. Zong N, Wong RSN, Yu Y, Wen A, Huang M, Li N. Drug-target prediction utilizing heterogeneous bio-linked network embeddings. Brief Bioinform 2021 Jan 18;22(1):568-580. [doi: 10.1093/bib/bbz147] [Medline: $\underline{31885036}$ ]

22. Pai S, Bader GD. Patient similarity networks for precision medicine. J Mol Biol 2018 Sep 14;430(18 Pt A):2924-2938 [FREE Full text] [doi: 10.1016/j.jmb.2018.05.037] [Medline: 29860027]

23. Bender D, Sartipi K. HL7 FHIR: an Agile and RESTful approach to healthcare information exchange. In: Proceedings of the 26th IEEE international symposium on computer-based medical systems. 2013 Presented at: IEEE international symposium on computer-based medical systems; June 20-22; Porto, Portugal. [doi: 10.1109/cbms.2013.6627810]

24. Grover A, Leskovec J. node2vec: scalable feature learning for networks. KDD 2016 Aug;2016:855-864 [FREE Full text] [doi: 10.1145/2939672.2939754] [Medline: 27853626]

25. Mandel JC, Kreda DA, Mandl KD, Kohane IS, Ramoni RB. SMART on FHIR: a standards-based, interoperable apps platform for electronic health records. J Am Med Inform Assoc 2016 Feb 17:899-908 [FREE Full text] [doi: 10.1093/jamia/ocv189] [Medline: 26911829]

26. Donnelly K. SNOMED-CT: The advanced terminology and coding system for eHealth. Stud Health Technol Inform 2006;121:279-290. [Medline: 17095826]

27. McDonald CJ, Huff SM, Suico JG, Hill G, Leavelle D, Aller R, et al. LOINC, a universal standard for identifying laboratory observations: a 5-year update. Clin Chem 2003 Apr;49(4):624-633 [FREE Full text] [Medline: 12651816]

28. International Statistical Classification of Diseases and Related Health Problems (ICD). World Health Organization. URL: http://www.who.int/classifications/icd/en/ [accessed 2021-05-11] 
29. Decker S, Melnik S, van Harmelen F, Fensel D, Klein M, Broekstra J, et al. The Semantic Web: the roles of XML and RDF. IEEE Internet Comput 2000;4(5):63-73. [doi: 10.1109/4236.877487]

30. Kaggal VC, Elayavilli RK, Mehrabi S, Pankratz JJ, Sohn S, Wang Y, et al. Toward a learning health-care system - knowledge delivery at the point of care empowered by big data and NLP. Biomed Inform Insights 2016;8(Suppl 1):13-22 [FREE Full text] [doi: 10.4137/BII.S37977] [Medline: 27385912]

31. Slee VN. The international classification of diseases: ninth revision (ICD-9). Ann Intern Med 1978 Mar 01;88(3):424. [doi: 10.7326/0003-4819-88-3-424]

32. Liu S, Wei Ma, Moore R, Ganesan V, Nelson S. RxNorm: prescription for electronic drug information exchange. IT Prof 2005 Sep;7(5):17-23. [doi: 10.1109/mitp.2005.122]

33. Hong N, Wen A, Shen F, Sohn S, Wang C, Liu H, et al. Developing a scalable FHIR-based clinical data normalization pipeline for standardizing and integrating unstructured and structured electronic health record data. JAMIA Open 2019 Dec;2(4):570-579 [FREE Full text] [doi: 10.1093/jamiaopen/ooz056] [Medline: 32025655]

34. Savova GK, Masanz JJ, Ogren PV, Zheng J, Sohn S, Kipper-Schuler KC, et al. Mayo clinical Text Analysis and Knowledge Extraction System (cTAKES): architecture, component evaluation and applications. J Am Med Inform Assoc 2010;17(5):507-513 [FREE Full text] [doi: 10.1136/jamia.2009.001560] [Medline: 20819853]

35. Sohn S, Clark C, Halgrim SR, Murphy SP, Chute CG, Liu H. MedXN: an open source medication extraction and normalization tool for clinical text. J Am Med Inform Assoc 2014;21(5):858-865 [FREE Full text] [doi: 10.1136/amiajnl-2013-002190] [Medline: 24637954]

36. Lin Y, Chen H, Brown RA. MedTime: a temporal information extraction system for clinical narratives. J Biomed Inform 2013 Dec;46 Suppl:S20-S28 [FREE Full text] [doi: 10.1016/j.jbi.2013.07.012] [Medline: 23911344]

37. Zong N, Sharma DK, Yu Y, Egan JB, Davila JI, Wang C, et al. Developing a FHIR-based framework for phenome wide association studies: a case study with a pan-cancer cohort. AMIA Jt Summits Transl Sci Proc 2020;2020:750-759 [FREE Full text] [Medline: $\underline{32477698]}$

38. O'Hara S, Draper B. Introduction to the bag of features paradigm for image classification and retrieval. arXiv. Preprint posted online on Jan 17, 2011.

39. Liaw A, Wiener M. Classification and regression by randomForest. R news 2002;2(3):18-22 [FREE Full text]

40. Huang Y, Li L. Naive Bayes classification algorithm based on small sample set. 2011 Presented at: IEEE International Conference on Cloud Computing and Intelligence Systems; September 15-17; Beijing, China. [doi: 10.1109/ccis.2011.6045027]

41. Zhou X, Liu K, Wong STC. Cancer classification and prediction using logistic regression with Bayesian gene selection. J Biomed Inform 2004 Aug;37(4):249-259 [FREE Full text] [doi: 10.1016/j.jbi.2004.07.009] [Medline: 15465478]

42. Noble WS. What is a support vector machine? Nat Biotechnol 2006 Dec;24(12):1565-1567. [doi: 10.1038/nbt1206-1565] [Medline: 17160063 ]

43. Liu W, Wang Z, Liu X, Zeng N, Liu Y, Alsaadi FE. A survey of deep neural network architectures and their applications. Neurocomputing 2017 Apr;234:11-26. [doi: 10.1016/j.neucom.2016.12.038]

44. Lawrence S, Giles CL, Tsoi AC, Back AD. Face recognition: a convolutional neural-network approach. IEEE Trans Neural Netw 1997;8(1):98-113. [doi: 10.1109/72.554195] [Medline: 18255614]

45. Kipf T, Welling M. Semi-supervised classification with graph convolutional networks. arXiv. Preprint posted online on February 22, 2017 [FREE Full text]

46. Azhagusundari B, Thanamani A. Feature selection based on information gain. Int J Innov Technol Explor Eng 2013;2(2):18-21 [FREE Full text]

47. node2vec. GitHub. URL: https://github.com/aditya-grover/node2vec [accessed 2011-05-11]

48. Machine Learning Group. LIBLINEAR -- a library for large linear classification. Taiwan University. URL: https://www. csie.ntu.edu.tw/ cjlin/liblinear/ [accessed 2021-05-11]

49. Weka. The University of Waikato. URL: https://www.cs.waikato.ac.nz/ml/weka/ [accessed 2021-05-10]

50. Chang CC, Line CJ. LIBSVM -- a library for support vector machines. Taiwan University. URL: https://www.csie.ntu.edu.tw/ cjlin/libsvm/ [accessed 2021-05-10]

51. Keras API reference. Keras. URL: https://keras.io/api/ [accessed 2021-05-10]

52. specktral. GitHub. URL: https://github.com/danielegrattarola/spektral [accessed 2021-05-10]

53. Davis J, Goadrich M. The relationship between precision-recall and ROC curves. In: Proceedings of the 23rd International Conference on Machine Learning. 2006 Presented at: 23rd International Conference on Machine Learning; June 25-29; Pittsburgh, Pennsylvania p. 233-240. [doi: 10.1145/1143844.1143874]

54. Nguyen G, Bouzerdoum A, Phung S. Learning pattern classification tasks with imbalanced data sets. In: Yin PY, editor. Pattern Recognition. London, United Kingdom: InTech Open; 2009:193-208.

55. Jeni LA, Cohn JF, De La Torre F. Facing imbalanced data recommendations for the use of performance metrics. Int Conf Affect Comput Intell Interact Workshops 2013;2013:245-251 [FREE Full text] [doi: 10.1109/ACII.2013.47] [Medline: 25574450]

56. Roc. GitHub. URL: https://github.com/kboyd/Roc [accessed 2021-05-10] 
57. Holmes G, Donkin A, Witten I. WEKA: a machine learning workbench. 1994 Presented at: Second Australian and New Zealand Conference on Intelligent Information Systems; November 29-December 2; Brisbane. [doi: 10.1109/anziis.1994.396988]

58. Woolson R. Wilcoxon signed-rank test. In: D'Agostino R, Massaro J, Sullivan L, editors. Wiley Encyclopedia of Clinical Trials. Hoboken, New Jersey: John Wiley \& Sons, Inc; 2007.

59. Lundberg SM, Lee SI. A unified approach to interpreting model predictions. 2017 Presented at: Advances in Neural Information Processing Systems 30; December 4-9; Long Beach, California.

60. Yang Y, Huang X, Zhou L, Deng T, Ning T, Liu R, et al. Clinical use of tumor biomarkers in prediction for prognosis and chemotherapeutic effect in esophageal squamous cell carcinoma. BMC Cancer 2019 May 31;19(1):526 [FREE Full text] [doi: 10.1186/s12885-019-5755-5] [Medline: 31151431]

61. Scarà S, Bottoni P, Scatena R. CA 19-9: biochemical and clinical aspects. Adv Exp Med Biol 2015;867:247-260. [doi: 10.1007/978-94-017-7215-0 15] [Medline: 26530370]

62. Høgdall E. Cancer antigen 125 and prognosis. Curr Opin Obstet Gynecol 2008 Feb;20(1):4-8. [doi: 10.1097/GCO.0b013e3282f2b124] [Medline: 18196998 ]

63. Zhang J, Huang T, Zhang F, Xu J, Chen G, Wang X, et al. Prognostic role of serum carbohydrate antigen 19-9 levels in patients with resectable hepatocellular carcinoma. Tumour Biol 2015 Apr;36(4):2257-2261. [doi: 10.1007/s13277-014-2435-6] [Medline: 25787748]

64. Wu H, Xiao J, Xiao S, Cheng Y. KRAS: a promising therapeutic target for cancer treatment. Curr Top Med Chem 2019;19(23):2081-2097. [doi: 10.2174/1568026619666190905164144] [Medline: 31486755]

65. Hankey W, Frankel WL, Groden J. Functions of the APC tumor suppressor protein dependent and independent of canonical WNT signaling: implications for therapeutic targeting. Cancer Metastasis Rev 2018 Mar;37(1):159-172 [FREE Full text] [doi: 10.1007/s10555-017-9725-6] [Medline: 29318445]

66. Du W, Lin C, Chen W. High expression of is an unfavorable prognostic biomarker in T4 gastric cancer patients. World J Gastroenterol 2019 Aug 21;25(31):4452-4467 [FREE Full text] [doi: 10.3748/wjg.v25.i31.4452] [Medline: $\underline{31496624}$ ]

67. Yassin A, AlRumaihi K, Alzubaidi R, Alkadhi S, Al Ansari A. Testosterone, testosterone therapy and prostate cancer. Aging Male 2019 Dec;22(4):219-227. [doi: 10.1080/13685538.2018.1524456] [Medline: 30614347]

68. Michaud JE, Billups KL, Partin AW. Testosterone and prostate cancer: an evidence-based review of pathogenesis and oncologic risk. Ther Adv Urol 2015 Dec;7(6):378-387 [FREE Full text] [doi: 10.1177/1756287215597633] [Medline: 26622322]

69. Hershman JM. Falling levels of thyroglobulin antibody after treatment for DTC predict no structural recurrence. Clin Thyroidol 2016 Mar;28(3):79-81. [doi: 10.1089/ct.2016;28.79-81]

70. Peiris AN, Medlock D, Gavin M. Thyroglobulin for monitoring for thyroid cancer recurrence. JAMA 2019 Mar 26;321(12):1228. [doi: 10.1001/jama.2019.0803] [Medline: 30912839]

71. Kim ES, Lim DJ, Baek KH, Lee JM, Kim MK, Kwon HS, et al. Thyroglobulin antibody is associated with increased cancer risk in thyroid nodules. Thyroid 2010 Aug;20(8):885-891. [doi: 10.1089/thy.2009.0384] [Medline: 20465529]

72. Santhanam P, Ladenson PW. Surveillance for differentiated thyroid cancer recurrence. Endocrinol Metab Clin North Am 2019 Mar;48(1):239-252. [doi: 10.1016/j.ecl.2018.11.008] [Medline: 30717906]

73. Jin $\mathrm{A}, \mathrm{Xu} \mathrm{J}$, Wang Y. The role of TERT promoter mutations in postoperative and preoperative diagnosis and prognosis in thyroid cancer. Medicine (Baltimore) 2018 Jul;97(29):e11548 [FREE Full text] [doi: 10.1097/MD.0000000000011548] [Medline: 30024548$]$

74. Li X, Dai D, Chen B, Tang H, Xie X, Wei W. Clinicopathological and prognostic significance of cancer antigen 15-3 and carcinoembryonic antigen in breast cancer: a meta-analysis including 12,993 patients. Dis Markers 2018;2018:9863092 [FREE Full text] [doi: 10.1155/2018/9863092] [Medline: 29854028]

75. Liu H, Xu Y, Xiang J, Long L, Green S, Yang Z, et al. Targeting alpha-fetoprotein (AFP)-MHC complex with CAR T-cell therapy for liver cancer. Clin Cancer Res 2017 Jan 15;23(2):478-488 [FREE Full text] [doi: 10.1158/1078-0432.CCR-16-1203] [Medline: 27535982]

76. Bethune G, Bethune D, Ridgway N, Xu Z. Epidermal growth factor receptor (EGFR) in lung cancer: an overview and update. J Thorac Dis 2010 Mar;2(1):48-51 [FREE Full text] [Medline: 22263017]

77. Widschwendter M, Jones A, Evans I, Reisel D, Dillner J, Sundström K, FORECEE (4C) Consortium. Epigenome-based cancer risk prediction: rationale, opportunities and challenges. Nat Rev Clin Oncol 2018 May;15(5):292-309. [doi: 10.1038/nrclinonc.2018.30] [Medline: 29485132]

78. Lee C, Peng C, Li R, Chen Y, Tsai H, Hung Y, et al. Risk evaluation for the development of cervical intraepithelial neoplasia: development and validation of risk-scoring schemes. Int J Cancer 2015 Jan 15;136(2):340-349 [FREE Full text] [doi: 10.1002/ijc.28982] [Medline: 24841989]

79. Teschendorff AE, Jones A, Fiegl H, Sargent A, Zhuang JJ, Kitchener HC, et al. Epigenetic variability in cells of normal cytology is associated with the risk of future morphological transformation. Genome Med 2012 Mar 27;4(3):24 [FREE Full text] [doi: 10.1186/gm323] [Medline: 22453031]

80. Machine learning repository. UCI. URL: https://archive.ics.uci.edu/ml/index.php [accessed 2021-05-14] 
81. Huang M, Chen C, Lin W, Ke S, Tsai C. SVM and SVM ensembles in breast cancer prediction. PLoS One 2017;12(1):e0161501 [FREE Full text] [doi: 10.1371/journal.pone.0161501] [Medline: 28060807]

82. Kumari M, Singh V. Breast cancer prediction system. Procedia Computer Science 2018;132:371-376. [doi: 10.1016/j.procs.2018.05.197]

83. Mostavi M, Chiu Y, Huang Y, Chen Y. Convolutional neural network models for cancer type prediction based on gene expression. BMC Med Genomics 2020 Apr 03;13(Suppl 5):44 [FREE Full text] [doi: 10.1186/s12920-020-0677-2] [Medline: 32241303]

84. Xiao Y, Wu J, Lin Z, Zhao X. A semi-supervised deep learning method based on stacked sparse auto-encoder for cancer prediction using RNA-seq data. Comput Methods Programs Biomed 2018 Nov;166:99-105. [doi: 10.1016/j.cmpb.2018.10.004] [Medline: 30415723]

85. Hou Q, Bing Z, Hu C, Li M, Yang K, Mo Z, et al. RankProd combined with genetic algorithm optimized artificial neural network establishes a diagnostic and prognostic prediction model that revealed C1QTNF3 as a biomarker for prostate cancer. EBioMedicine 2018 Jun;32:234-244 [FREE Full text] [doi: 10.1016/j.ebiom.2018.05.010] [Medline: 29861410]

86. Liu J, Wang X, Cheng Y, Zhang L. Tumor gene expression data classification via sample expansion-based deep learning. Oncotarget 2017 Dec 12;8(65):109646-109660 [FREE Full text] [doi: 10.18632/oncotarget.22762] [Medline: 29312636]

87. Miotto R, Li L, Kidd BA, Dudley JT. Deep Patient: an unsupervised representation to predict the future of patients from the electronic health records. Sci Rep 2016 Dec 17;6:26094 [FREE Full text] [doi: 10.1038/srep26094] [Medline: 27185194]

88. Wang X, Zhang Y, Hao S, Zheng L, Liao J, Ye C, et al. Prediction of the 1-year risk of incident lung cancer: prospective study using electronic health records from the State of Maine. J Med Internet Res 2019 May 16;21(5):e13260 [FREE Full text] [doi: 10.2196/13260] [Medline: $\underline{31099339]}$

89. Wu Y, Burnside E, Cox J, Fan J, Yuan M, Yin J. Breast cancer risk prediction using electronic health records. 2017 Presented at: IEEE International Conference on Healthcare Informatics; August 23-36; Park City, Utah. [doi: 10.1109/ichi.2017.62]

90. Muhammad W, Hart GR, Nartowt B, Farrell JJ, Johung K, Liang Y, et al. Pancreatic cancer prediction through an artificial neural network. Front Artif Intell 2019 May 3;2(2):1. [doi: 10.3389/frai.2019.00002]

91. Wan J, Chen B, Kong Y, Ma X, Yu Y. An early intestinal cancer prediction algorithm based on deep belief network. Sci Rep 2019 Nov 22;9(1):17418 [FREE Full text] [doi: 10.1038/s41598-019-54031-2] [Medline: $\underline{31758076]}$

92. Pavlidis N, Pentheroudakis G. Cancer of unknown primary site. Lancet 2012 Apr 14;379(9824):1428-1435. [doi: 10.1016/S0140-6736(11)61178-1] [Medline: 22414598]

93. Mikolov T, Karafiát M, Burget L, ?ernocký J, Khudanpur S. Recurrent neural network based language model. 2010 Presented at: Eleventh Annual Conference of the International Speech Communication Association; September 26-30; Makuhari, Japan.

94. Gers FA, Schmidhuber J, Cummins F. Learning to forget: continual prediction with LSTM. Neural Comput 2000 Oct;12(10):2451-2471. [doi: 10.1162/089976600300015015] [Medline: 11032042]

95. Hripcsak G, Duke JD, Shah NH, Reich CG, Huser V, Schuemie MJ, et al. Observational Health Data Sciences and Informatics (OHDSI): opportunities for observational researchers. Stud Health Technol Inform 2015;216:574-578 [FREE Full text] [Medline: 26262116]

96. cancer-prediction-on-fhir-rdf. GitHub. URL: https://github.com/fhircat/cancer-prediction-on-fhir-rdf [accessed 2021-05-10]
Abbreviations
AUPRC: area under the precision-recall curve
AUROC: area under the receiver operating characteristic curve
ICD: International Statisitical Classification of Diseases
FHIR: Fast Healthcare Interoperability Resources
RDF: Resource Description Framework
ReLU: rectified linear unit

Edited by G Eysenbach; submitted 17.08.20; peer-reviewed by JF Silva, X Zhou; comments to author 13.11.20; revised version received
07.01.21; accepted 27.01.21; published 25.05.21
Please cite as:
Zong N, Ngo V, Stone DJ, Wen A, Zhao Y, Yu Y, Liu S, Huang M, Wang C, Jiang G
Leveraging Genetic Reports and Electronic Health Records for the Prediction of Primary Cancers: Algorithm Development and
Validation Study
JMIR Med Inform 2021;9(5):e23586
URL: https:///medinform.jmir.org/2021/5/e23586
doi: $10.2196 / 23586$
PMID:


(CNansu Zong, Victoria Ngo, Daniel J Stone, Andrew Wen, Yiqing Zhao, Yue Yu, Sijia Liu, Ming Huang, Chen Wang, Guoqian Jiang. Originally published in JMIR Medical Informatics (https://medinform.jmir.org), 25.05.2021. This is an open-access article distributed under the terms of the Creative Commons Attribution License (https://creativecommons.org/licenses/by/4.0/), which permits unrestricted use, distribution, and reproduction in any medium, provided the original work, first published in JMIR Medical Informatics, is properly cited. The complete bibliographic information, a link to the original publication on https://medinform.jmir.org/, as well as this copyright and license information must be included. 